\title{
Space telescope faces cuts
}

\section{NASA takes a hard look at capabilities of planned dark-energy probe as costs rise.}

\section{BY ALEXANDRA WITZE}

$\mathrm{N}$ ASA's next major space observatory is meant to tackle some of the biggest questions in astronomy when it launches in 2025 - including what exoplanets look like and how dark energy is driving the Universe's expansion. But the project's cost is rising quickly, and NASA managers are struggling to keep its budget in check.

The Wide-Field Infrared Survey Telescope (WFIRST) has grown in scope and complexity since it was proposed nearly a decade ago, and its price has swollen from US $\$ 1.6$ billion in 2010 to the current estimate of $\$ 3.2$ billion ( $\$ 2.4$ billion in 2010 dollars). That has raised concern at NASA, which in April commissioned a review by independent aerospace experts. Their report is due in the next few months.

Above all, the agency wants to keep WFIRST from following the path of the James Webb Space Telescope (JWST), a successor to the Hubble telescope that is scheduled to launch in 2018. That project's cost spiralled from $\$ 1$ billion in the early 2000 s to $\$ 8.8$ billion and nearly exhausted NASA's astrophysics budget.

The WFIRST review is meant to stave off that kind of meltdown. "This is a good time to take a look at the scale and scope of the mission," says Jon Morse, a former head of NASA's astrophysics division who is now chief executive of the BoldlyGo Institute, a non-profit spaceexploration organization in New York City. "Nobody wants this thing to double in cost."

\section{BONUS FEATURES}

WFIRST was the top-ranked big space mission in the 2010 decadal survey in astronomy and astrophysics, a list created by researchers to prioritize projects for the next ten years. Then the National Reconnaissance Office gave NASA a 2.4-metre mirror - replacing WFIRST's planned 1.5-metre mirror - and the space agency started dreaming big. The larger mirror allowed NASA to add a coronagraph, an instrument that studies exoplanets by blocking light from the stars they orbit.

And NASA made other design changes to go along with the big mirror. It also began to consider adding a 'starshade', a free-floating umbrella-like spacecraft that would fly alongside WFIRST and block enough light for the telescope to spy Earth-sized planets.

WFIRST's heart is a gigantic camera with 18 detectors, each capable of capturing a 16-megapixel shot - giving it a field of view

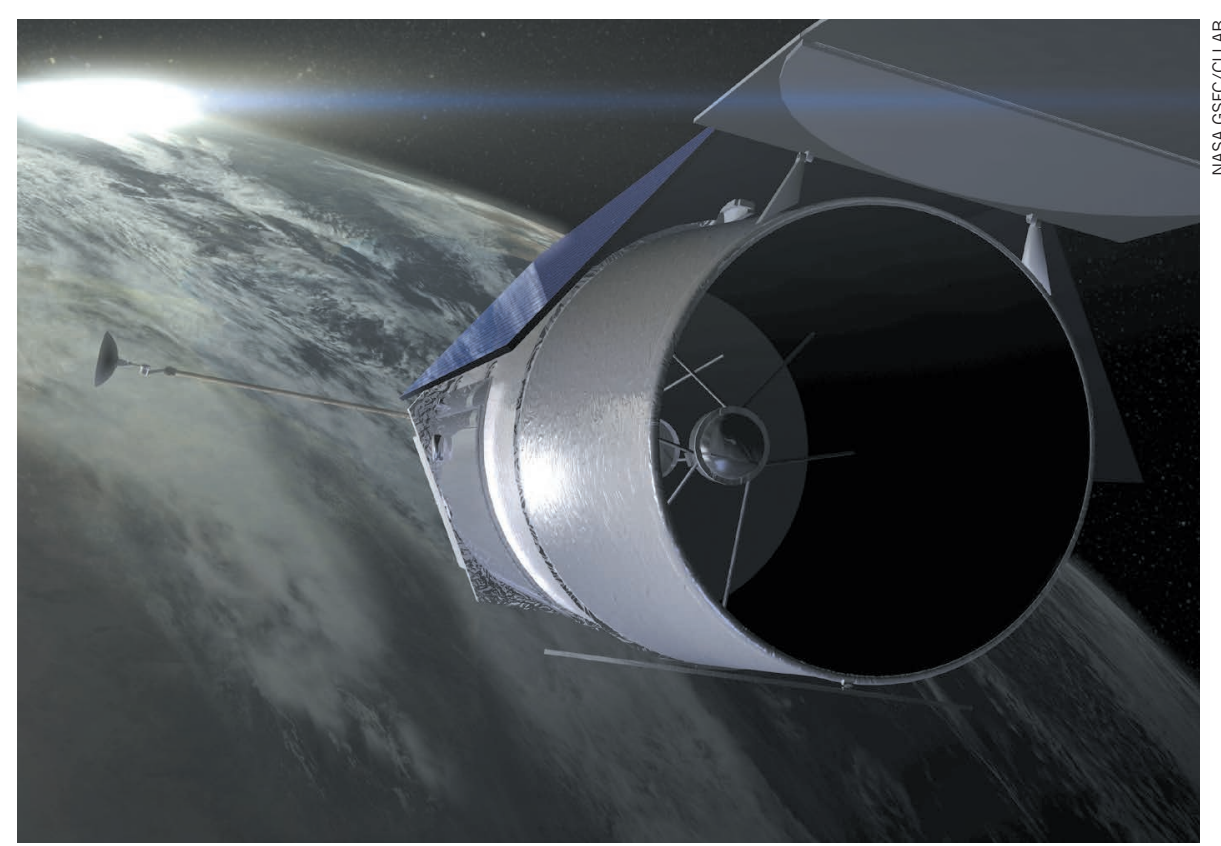

An illustration of the Wide-Field Infrared Survey Telescope, which is scheduled to launch in 2025.

200 times Hubble's. "When you have this enormous field of view you can address scientific problems that really are not practical with missions like Hubble or Webb," says Jeffrey Kruk, the WFIRST project scientist at the Goddard Space Flight Center in Greenbelt, Maryland.

Those include a survey to measure how the structure of the Universe evolved over time, which will shed light on the nature of dark energy. WFIRST's data should complement the observations of several other dark-energy explorers set to come online in the early 2020s, such as the European Space Agency's Euclid probe, says Rachel Mandelbaum, an astrophysicist at Carnegie Mellon University in Pittsburgh, Pennsylvania.

WFIRST's exoplanet studies will include hunting indirectly for planets in the bulge of stars at the centre of the Milky Way, and imaging others directly using the coronagraph. The coronagraph is meant to demonstrate technologies for future missions, but should also be able to photograph Neptune-sized planets. "We really hope and expect to do revolutionary exoplanet science," says Jeremy Kasdin, a technologist and engineer at Princeton University in New Jersey who leads the coronagraph team.

But there is only so much money to put towards all these goals. Last August, a review of NASA's progress towards its 2010 decadal priorities singled out WFIRST as at risk of ballooning costs. The review cited the cost of the coronagraph - which a different panel estimated at around \$350 million — and design changes that added another \$550 million.

The new study will help NASA evaluate how to preserve as much of WFIRST's scientific capability as possible while remaining within budget, says John Gagosian, the mission's programme executive at NASA headquarters in Washington DC. But he sees no reasonable scenario "in which the current mission scope and requirements (including the coronagraph) can be implemented for $\$ 3.2$ billion or less".

One potential cut would be to eliminate the coronagraph or to pare back its capabilities. Another would be to trim the number of detectors on the wide-field camera, or the amount of time dedicated to the dark-energy survey.

Whether such belt-tightening is enough to keep WFIRST under $\$ 3.2$ billion is unclear. A way to save money year-to-year would be to stretch the project's lifespan, says Kruk — but that increases the total cost. And launching it later than 2025 would cut back on the mission's chance to overlap with the JWST and find rare celestial objects that that telescope could then study in detail.

The next major milestone for WFIRST will come after the review panel submits its recommendations. Late this year or early next, programme managers will decide what they may need to strip off the spacecraft to keep the project alive. 